\title{
Analysis of Double-Substrate Limited Growth
}

\author{
F. G. BADER, * Department of Chemical Engineering, University \\ of Michigan, Ann Arbor, Michigan 48109
}

\begin{abstract}
Summary
Mathematical models which relate the growth rate of a microorganism to a single limiting substrate concentration have long been established. In recent years, it has become apparent that, under certain conditions, the growth rate of an organism may be simultaneously limited by two or more substrates. Mathematical models of double-substrate limitation fall into two categories: interactive and noninteractive models. A discussion of both types of models is presented in both conceptual and mathematical terms. An analogous case of an enzyme which requires two different substrates to produce a single product is presented. This enzyme analog indicates that both types of double-substrate limitation models appear to be feasible under certain conditions. Based upon stoichiometry and specific growth rate-substrate concentration contour plots, a method for determining the operational conditions which will lead to double-substrate limitation is presented.
\end{abstract}

\section{INTRODUCTION}

Mathematical models which describe the growth rate of a biological population as a function of a single limiting substrate have existed, at least since Blackman published his model in $1905 .^{1}$ These models provided a necessary theoretical tool which has aided in understanding many natural biological systems and in developing industrial processes. In recent years it has become apparent that the possibility exists that at least some natural or industrial biological populations may be simultaneously limited by more than one substrate. A new concept has also developed: that one substrate may control the rate of growth while another may ultimately control the extent of growth.

From a theoretical viewpoint, it would be desirable to develop a general mathematical model which handles all types of doublesubstrate limitation. This becomes rather difficult since two separate schools of thought exist about the nature of growth with two limiting substrates, and there is insufficient experimental data to

\footnotetext{
* Current address: The Upjohn Company, Unit 1500, Kalamazoo, MI 49001.
}

Biotechnology and Bioengineering Vol. XX, Pp. 183-202 (1978)

(C) 1978 John Wiley \& Sons, Inc.

0006-3592/78/0020-0183\$01.00 
support either school. In fact, it is doubtful that sufficient experimental evidence will be developed in the near future.

The present paper is a follow-up to a previously published paper. ${ }^{2}$ The purpose is to further develop the two types of models which may be used for describing double-substrate limited growth. Double-substrate limitation is not likely to occur readily in most real systems. The conditions under which it is likely to occur are discussed in both conceptual and theoretical forms.

\section{BASIC SINGLE-SUBSTRATE MODELS}

Many different types of single-substrate limited growth models exist. The choice of model often depends upon the type of organism being studied. For example, light-limited algal growth models are slightly different than bacterial models, which in turn may be different from zooplankton models. Most models generally degenerate to one of three forms which can be represented by the Monod, ${ }^{3}$ Blackman, ${ }^{1}$ and exponential kinetic models. These are in a dimensionless form: Monod

$$
\frac{\mu}{\mu_{m}}=\frac{S_{1} / K_{1}}{1+S_{1} / K_{1}}
$$

Blackman

$$
\begin{aligned}
& \frac{\mu}{\mu_{m}}=\frac{1}{2} \frac{S_{1}}{K_{1}} \quad \text { for } S_{1}<2 K_{1} \\
& \frac{\mu}{\mu_{m}}=1 \quad \text { for } S_{1}>2 K_{1}
\end{aligned}
$$

exponential

$$
\frac{\mu}{\mu_{m}}=\left(1-\exp \left\{-0.6931 \frac{S_{1}}{K_{1}}\right\}\right)
$$

In these models, $\mu_{m}$ is the maximum specific growth rate of the organism, $\mu$ is the actual specific growth rate of the organism at a limiting substrate concentration equal to $S_{1}$, and $K_{1}$ is the half velocity constant (equal to the substrate concentration at which the organism grows at half its maximum rate). A plot of the three models is shown in Figure 1. The dots in Figure 1 represent typical experimental results. A similar comparison, using real data, has been presented by Dabes et al. ${ }^{4}$ 


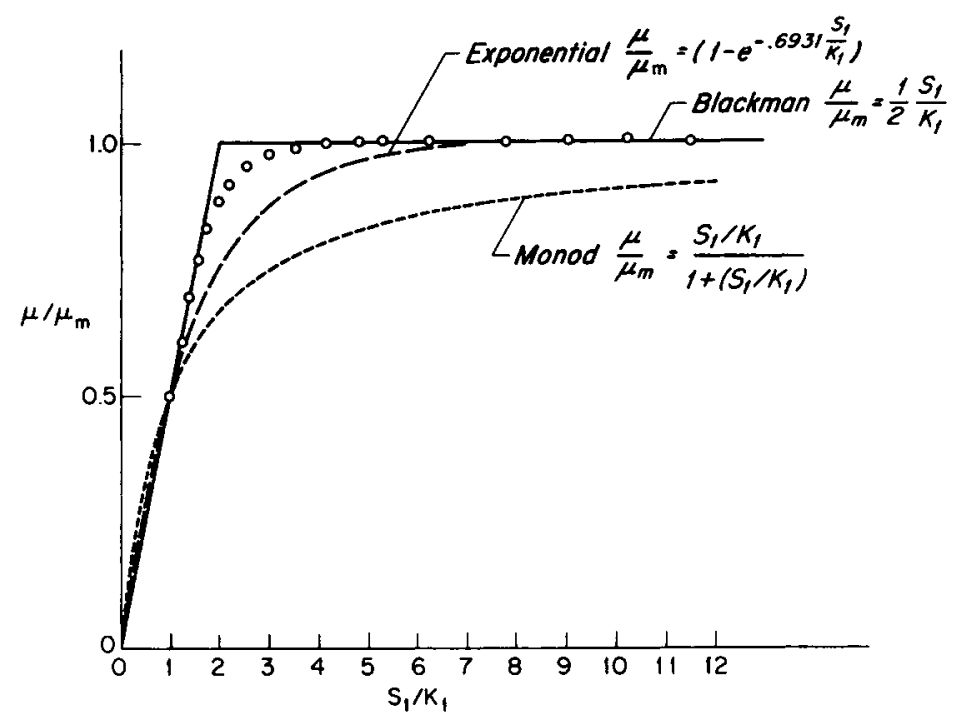

Fig. 1. Dimensionless plot of the three basic models for substrate limited growth of bacterial cells. Models have been normalized so that $\mu / \mu_{m}=0.5$ at $S_{1} / K_{1}=1$. Circles represent typical experimental results.

The Monod model is the most commonly used of the three models. It is equivalent to the Michaelis-Menten enzyme kinetic model which is covered in many basic biology and chemistry texts. The Monod model is also a simple and continuous algebraic relationship which lends itself readily to theoretical analysis. Of the three models, the Monod model generally represents the worst fit to experimental data. As can be seen in Figure 1, it does not saturate rapidly enough at higher substrate concentrations.

The Blackman and exponential models generally give a better fit to experimental data; primarily because they both saturate faster than the Monod model. Most data fall between the Blackman and exponential models as shown in Figure 1. Both of these models are not frequently used because of the discontinuity in the Blackman model and the exponential term in the exponential model. These lead to mathematical complexities which one frequently would prefer to avoid.

An organism that is growing at its maximum rate is said to be saturated with substrate, i.e., addition of more substrate would not increase its growth rate. Of the three models, only the Blackman model truly saturates. Both the Monod and exponential models asymptotically approach the maximum growth rate $\left(\mu / \mu_{m}=1\right)$. 


\section{DOUBLE-SUBSTRATE MODELS}

When an organism is growing in an environment in which two of its required substrates are present at less than saturating levels, double-substrate limitation may occur. Two different philosophies have developed concerning the growth rate of the organism under

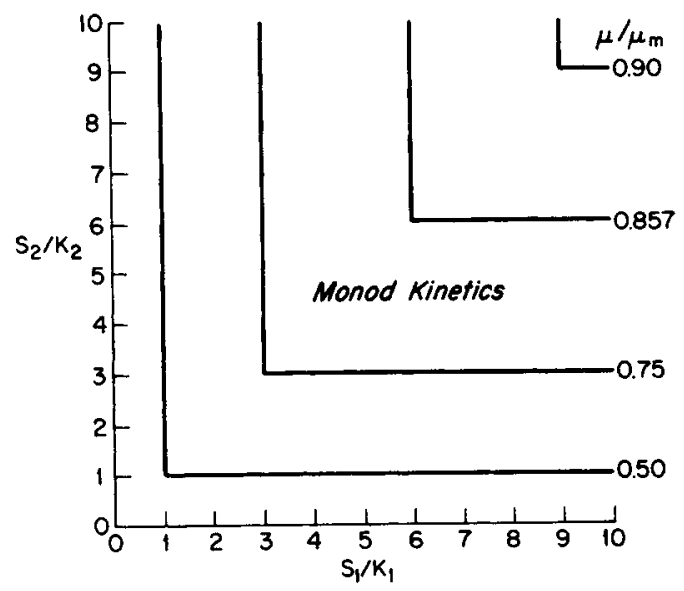

(a)

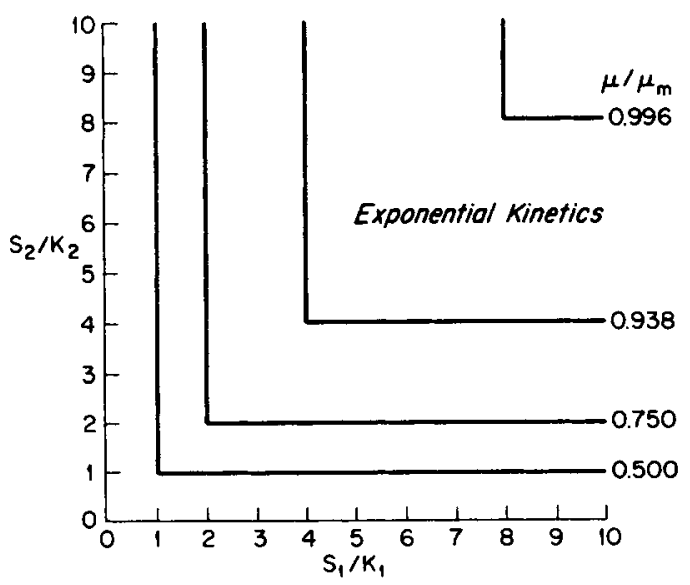

(b)

Fig. 2. Plots of lines of constant dimensionless specific growth rate $\mu / \mu_{m}$ as a function of two dimensionless substrate concentrations for noninteractive models using (a) Monod kinetics, (b) exponential kinetics, and (c) Blackman kinetics. Note that a dimensionless specific growth rate of $\mu / \mu_{m}=1$ is only attained with Blackman kinetics. 
this type of condition. These may be classified as interactive and noninteractive double-substrate limited growth models.

\section{Noninteractive Model}

A noninteractive model basically implies that the growth rate of the organism can only be limited by one substrate at a time. Therefore, the growth rate of the organism will be equal to the lowest growth rate that would be predicted from the separate single-substrate models. For the Monod model, this may be written

$$
\begin{array}{rlrl}
\frac{\mu}{\mu_{m}} & =\frac{S_{1} / K_{1}}{1+S_{1} / K_{1}} & & \frac{S_{1}}{K_{1}}<\frac{S_{2}}{K_{2}} \\
\frac{\mu}{\mu_{m}}=\frac{S_{2} / K_{2}}{1+S_{2} / K_{2}} & \frac{S_{2}}{K_{2}}<\frac{S_{1}}{K_{1}}
\end{array}
$$

Similar equations may be written for Blackman and exponential kinetics. Graphs of constant dimensionless growth rate $\left(\mu / \mu_{m}\right)$ as a function of dimensionless substrate concentrations $\left(S_{1} / K_{1}\right.$ and $S_{2} /$ $K_{2}$ ) are shown for all three types of kinetics in Figure 2. Examples of this type of approach may be found in articles by Sykes, ${ }^{5}$ Droop, ${ }^{6}$ and Ryder and Sinclair. ${ }^{7}$

The rationale for noninteractive models may be developed using the schematic model shown in Figure 3. Consider an organism which has two separate subsystems (biochemical pathways) that consume different substrates and produce different products which

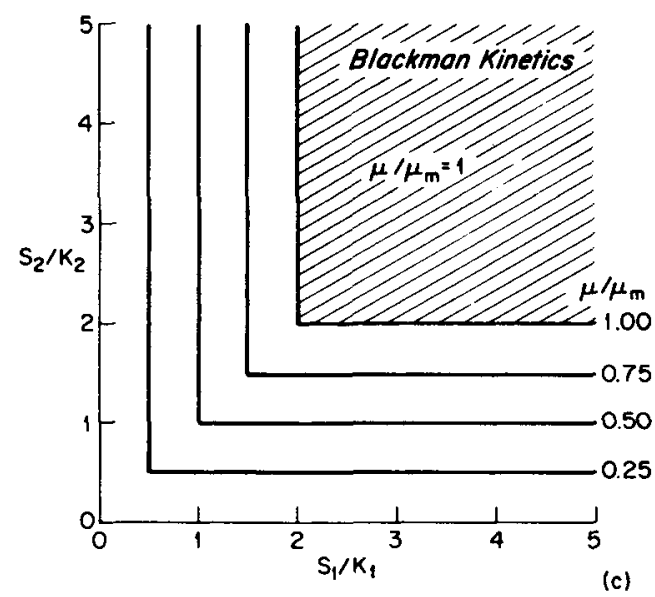

Fig. 2. (Continued from previous page.) 


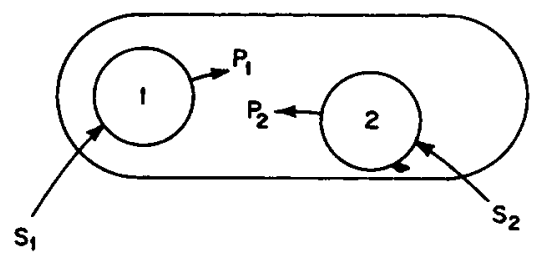

Fig. 3. Conceptual representation of the noninteractive model. Systems 1 and 2 operate independently of one another.

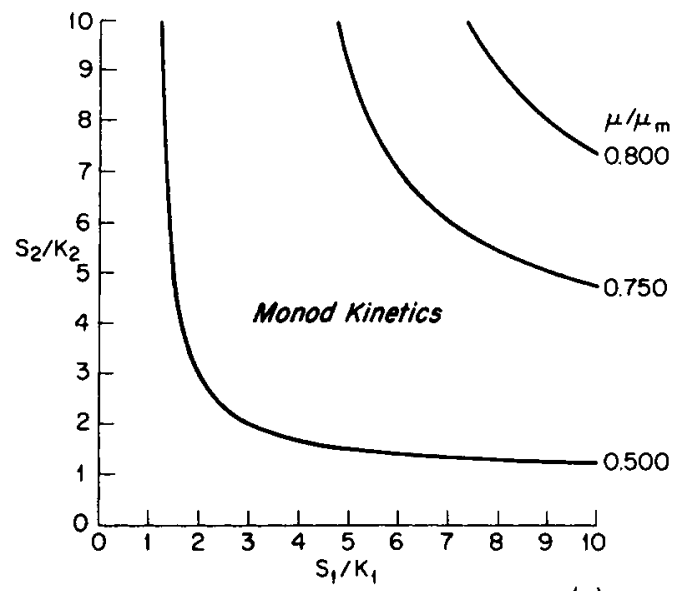

(a)

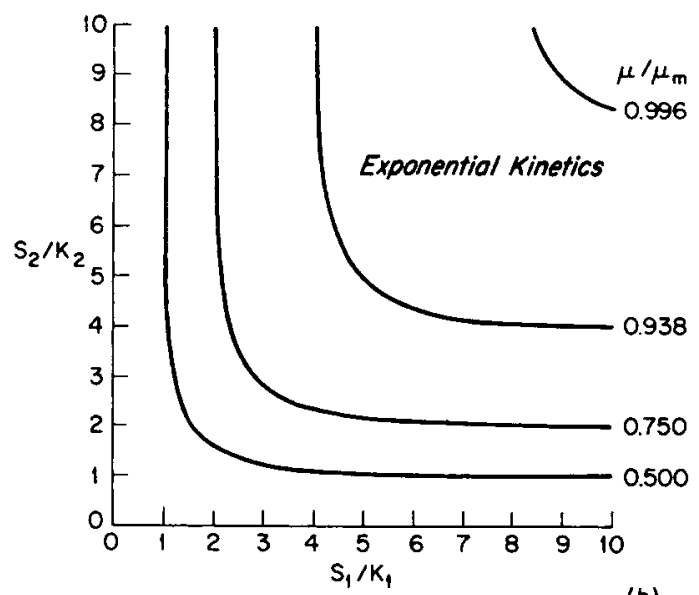

(b)

Fig. 4. Plots of lines of constant dimensionless specific growth rate $\mu / \mu_{m}$ as a function of two dimensionless substrate concentrations for interactive models of the McGee-type, (a) Monod, (b) exponential, and (c) Blackman kinetics. 


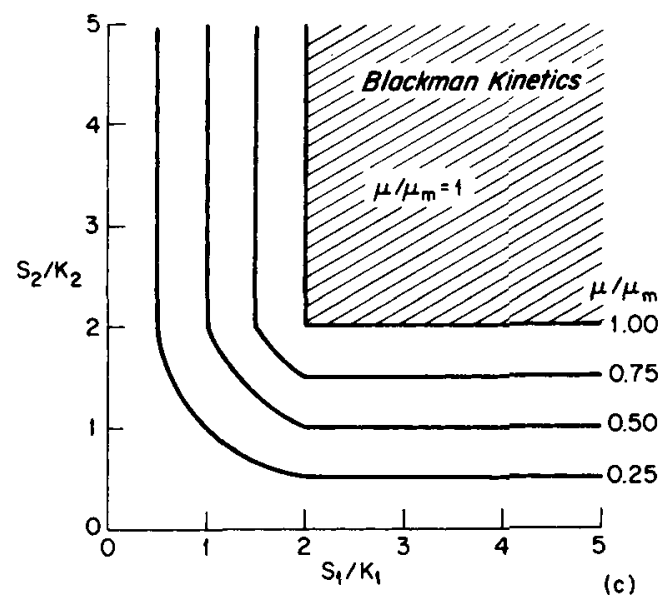

Fig. 4. (Continued from previous page.)

are required for the growth of the organism. If the two subsystems do not require intermediates or products from one another, they can function independently. If the substrate concentrations $S_{1}$ and $S_{2}$ are such that subsystem 1 and 2 are producing their products at one-half and one-fourth of their maximum rates, respectively, then product 1 would be present in excess and only product 2 would limit the growth rate of the organism. The growth rate of the organism would be one-fourth its maximum growth rate, or limited only by substrate 2 .

Since the general physiological state of an organism depends upon the availability of all nutrients, it is unlikely that any two cellular subsystems would be totally independent of each other. However, the degree of interaction between certain subsystems may be rather small. In such cases, a noninteractive-type of model may accurately describe the growth rate of the organism.

\section{Interactive Models}

An interactive model is based upon the assumption that if two substrates are present in less than saturating concentrations, then both must affect the overall growth rate of the organism. The simplest type of interactive model may be constructed by simply multiplying two single-substrate limited models together,

$$
\frac{\mu}{\mu_{m}}=\frac{S_{1} / K_{1}}{1+S_{1} / K_{1}} \frac{S_{2} / K_{2}}{1+S_{2} / K_{2}}
$$


This particular model was developed by McGee et al. ${ }^{8}$ and has been subsequently used by Ryder and Sinclair $^{7}$ and Howell and Atkinson. ${ }^{9}$ Similar equations may be written for the Blackman and exponential type of kinetics. Plots of these models are shown in Figure 4.

Two cases which provide a rational basis for the interactive model are shown in Figures 5(a) and 5(b). In the first case (5a) a cell has a certain number of enzymes present which, in the presence of a cofactor $\left(S_{2}\right)$, convert a substrate $\left(S_{1}\right)$ to a product $\left(P_{1}\right)$ which is required for the growth of the cell. If the external substrate $\left(S_{1}\right)$ and cofactor $\left(S_{2}\right)$ concentrations are both at half velocity levels, then only one-half of the total enzyme would be active, and the active enzyme would be producing product at onehalf its maximum rate. The overall rate of $P_{1}$ production and the growth rate of the cell would be one-fourth the maximum possible rate. This agrees with the prediction of the McGee-type model.

In the second case (Fig. 5(b)) two substrates $\left(S_{1}\right.$ and $\left.S_{2}\right)$, or their derivatives, are required to produce a single product $\left(P_{1}\right)$, which is required for the growth of the cell. This is probably the most common type of interaction between substrates which are required for the anabolic functions of the organism. The relationship between the product production rate and the two substrate concen-
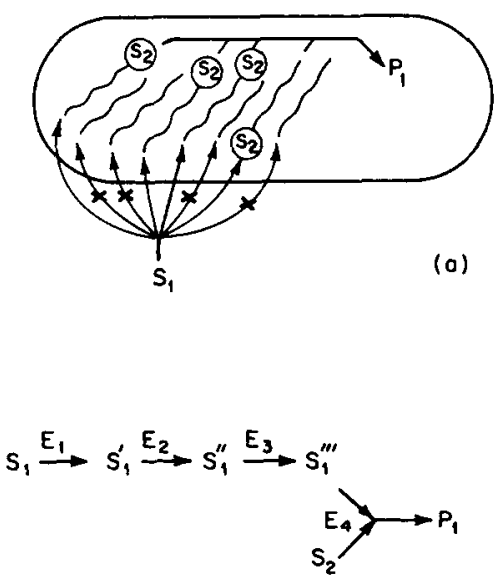

(b)

Fig. 5. Conceptual representation of the interactive model. (a) $S_{1}$ is converted to $P_{1}$ by an enzyme which requires $S_{2}$ as a cofactor. (b) Substrates $S_{1}$ and $S_{2}$ from two parallel pathways are combined by an enzyme $E_{T}$ to produce a product $P_{1}$ which is required for growth. 
trations is not readily self-evident for this system. However, it can be derived from basic enzyme kinetics.

\section{TWO-SUBSTRATE ENZYME MODEL}

Consider the system shown in Figure 6 where we have a single enzyme which must pick up two different substrates to combine them into a single product. In theory, this can happen by either route 1 or route 2 depending upon which substrate complexes with the enzyme first. It should be recognized that it is possible that only one route may actually occur owing to allosteric properties of the enzyme. Three possible cases exist for this system: only route 1 operates, only route 2 operates, and both routes operate. The first two cases are rather simple and are shown in Appendix I.

By the analogy shown in the bottom of Appendix I, we can use the result from the single route enzyme system to describe the growth rate of the organism. Equations (19) and (20) (Appendix I) are similar in form to the McGee model (eq. (5)) and are identical to the McGee model if $\alpha^{\prime}$, or $\alpha^{\prime \prime}$ is equal to one. A dimensionless plot of the single route equations for a dimensionless growth rate $\left(\mu / \mu_{m}\right)$ equal to 0.5 and for various values of $\alpha$ is shown in Figure 7.

The analysis of the system, where both routes are acting simultaneously, is shown in Appendix II. There are nine separate rate constants for this system for which values must be set. The single route analysis has produced relationships for the half velocity constants if either route dominates. If the half velocity constants for single-substrate limited growth are known for both

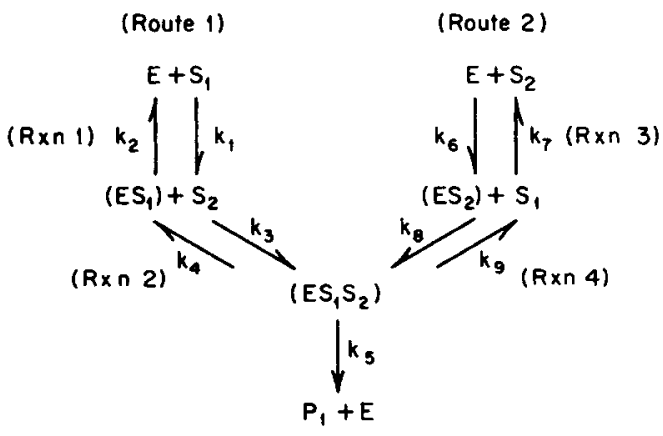

Fig. 6. Two-substrate enzyme reactions. Substrates $S_{1}$ and $S_{2}$ are combined to form a single product $P_{1}$. This set of reactions are analyzed in Appendices I and II. 


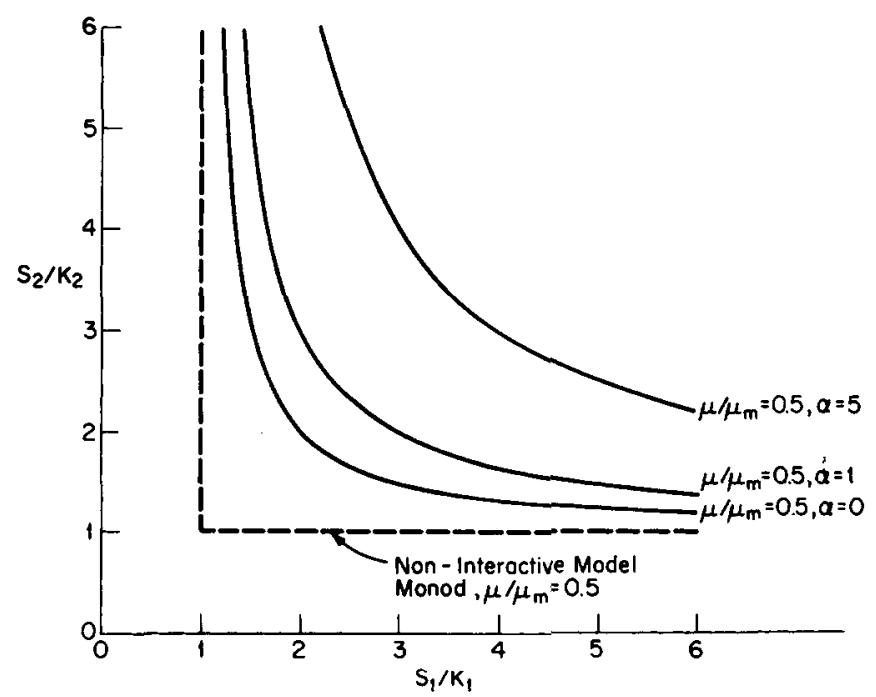

Fig. 7. Plot of the single route-double-substrate enzyme equations shown in Appendix I. Lines of $\mu / \mu_{m}=0.5$ are plotted as a function of dimensionless substrate concentration for different values of $\alpha$. McGee model is equivalent to $\alpha=1$. Dashed line is the noninteractive model using Monod kinetics.

substrates, then these can be used to evaluate some of the individual rate constants:

$$
\begin{aligned}
& k_{1}=k_{5} / K^{\prime} \\
& k_{3}=\left(k_{4}+k_{5}\right) / K_{2} \\
& k_{6}=k_{5} / K^{\prime}{ }_{2} \\
& k_{8}=\left(k_{9}+k_{5}\right) / K_{1}
\end{aligned}
$$

Note that $k_{5}$ may have any value as it drops out when analyzing for the dimensionless growth rate $\left(\mu / \mu_{m}\right)$. There are now four rate constants $\left(k_{2}, k_{4}, k_{7}, k_{9}\right)$ which must be assigned values. A relative handle may be obtained for these by looking at the equilibrium relationships for each of the reactions:

$$
\begin{array}{ll}
k_{1} / k_{2}=X_{1} & k_{6} / k_{7}=X_{3} \\
k_{3} / k_{4}=X_{2} & k_{8} / k_{9}=X_{4}
\end{array}
$$

All of the rate constants can be evaluated by specifying values for $X_{1}-X_{4}$. Values of $X$ greater than one indicate a forward reaction equilibrium and values less than one indicate a reverse reaction 
equilibrium. A computer solution of eqs. (21)-(24) has been conducted where values of $X$ were limited to 10 (forward reaction equilibrium) and 0.1 (reverse reaction equilibrium). Since we have four $X$ 's, all possible combinations of two values for the four $X$ 's give us 16 separate solutions for a given value of dimensionless holding time which was specified as $\mu / \mu_{m}=0.5$.

The results of the computer solution are shown in Figure 8. Only five of the 16 curves are shown as the remaining curves are either identical or very similar to those that are shown. Curves of type $A$ and $\mathrm{B}$ in Figure 8 are rather close approximations to the noninteractive model discussed earlier. These curves occur when the equilibrium of both reactions (2) and (4) (i.e., $X_{2}$ and $X_{4}$ ) have a forward reaction equilibrium. Curve $\mathrm{E}$ shows the highest level of involvement of both substrates in the overall operating rate of the system. This curve occurs when both reactions (2) and (4) have a reverse reaction equilibrium. Curves $C$ and $D$ are intermediate cases which result when either reaction (2) or (4) has a forward equilibrium, while the other has a reverse equilibrium. Curve $D$ is identical to the prediction of the McGee model.

The two-substrate enzyme system indicates that a range in the level of interaction between the two substrates may occur, depending upon the relative values of the particular rate constants for the system. It does not invalidate the potential use of either the

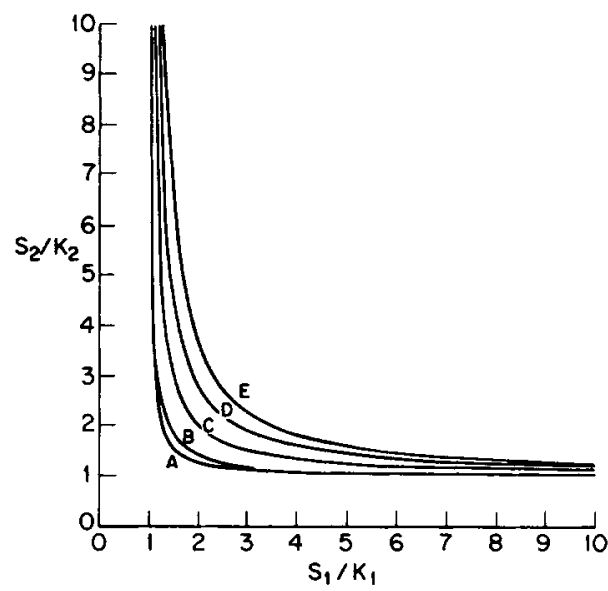

Fig. 8. Results of the double-substrate enzyme model (Fig. 6) shown in Appendix II where both routes are operating. All curves are for $\mu / \mu_{m}=0.5$. See text for explanation of the different curves. 
noninteractive or the interactive model (eqs. (4) and (5)) in describing such systems. Rather, the two-substrate enzyme system indicates that a unique model, which would describe all cases of twosubstrate limited growth, is not likely to exist. Each case of doublesubstrate limitation may exert slightly different behavior. Since good experimental evidence is not available for double-substrate limited growth systems, the most convenient kinetic model is probably the best initial choice.

\section{CONDITIONS FOR DOUBLE-SUBSTRATE LIMITATION}

The general differential equations which describe a cell population $\left(b_{1}\right)$ which is growing on two potentially limiting substrates may be written in a general form as follows:

$$
\begin{aligned}
& \frac{d b_{1}}{d t}=\mu_{m} f\left(S_{1}, S_{2}\right) b_{1}-\frac{b_{1}}{\theta} \\
& \frac{d S_{1}}{d t}=-\frac{\mu_{m}}{Y_{1}} f\left(S_{1}, S_{2}\right) b_{1}+\frac{S_{f_{1}}-S_{1}}{\theta} \\
& \frac{d S_{2}}{d t}=-\frac{\mu_{m}}{Y_{2}} f\left(S_{1}, S_{2}\right) b_{1}+\frac{S_{f_{2}}-S_{2}}{\theta}
\end{aligned}
$$

where $f\left(S_{1}, S_{2}\right)$ is the relationship between the growth rate and the two substrate concentrations, $\theta$ is the holding time for the system (hr), $Y_{1}$ and $Y_{2}$ are the yield coefficients for substrate $S_{1}$ and $S_{2}$, respectively (cells/g substrate), and $S_{f_{1}}$ and $S_{f_{2}}$ are the feed concentrations of the two substrates ( $\mathrm{g} /$ liter).

At steady state, the concentration of organisms is given by:

$$
\tilde{b}_{1}=Y_{1}\left(S_{f_{1}}-\tilde{S}_{1}\right)=Y_{2}\left(S_{f_{2}}-\tilde{S}_{2}\right)
$$

where the tilde represents steady-state values. Equation (10) is a stoichiometric relationship. This equation may be rearranged in terms of dimensionless substrate concentrations as follows:

$$
\tilde{b}_{1}=Y_{1} K_{1}\left[\frac{S_{f_{1}}}{K_{1}}-\frac{\tilde{S}_{1}}{K_{1}}\right]=Y_{2} K_{2}\left[\frac{S_{f_{2}}}{K_{2}}-\frac{\tilde{S}_{2}}{K_{2}}\right]
$$

If we let:

$$
\begin{array}{ll}
\alpha_{f}=S_{f_{1}} / K_{1} & \tilde{\alpha}=\tilde{S}_{1} / K_{1} \\
\beta_{f}=S_{\delta_{2}} / K_{2} & \tilde{\beta}=\tilde{S}_{2} / K_{2}
\end{array}
$$


then clearly

$$
\beta_{f}-\tilde{\beta}=\left(Y_{1} K_{1} / Y_{2} K_{2}\right)\left(\alpha_{f}-\tilde{\alpha}\right)
$$

which defines a dimensionless stoichiometric line which relates the steady-state values of the substrates.

For the growth rate of an organism to be able to switch between which substrate is controlling, or to have both substrates controlling simultaneously, the stoichiometric line must intersect the transition line, $\alpha=\beta$, as shown in Figure 9. (Lines of constant specific growth rate $\mu / \mu_{m}$ may be plotted on this figure for any of the six kinetic models discussed, similar to Figs. 2 and 4.) The point of intersection $\left(\alpha_{I}\right)$ may be obtained by setting $\alpha_{I}=\bar{\alpha}=\bar{\beta}$ in the stoichiometric equation and solving:

$$
\alpha_{I}=\frac{\left(Y_{1} K_{1} / Y_{2} K_{2}\right) \alpha_{f}-\beta_{f}}{\left(Y_{1} K_{1} / Y_{2} K_{2}\right)-1}
$$

Note that $\alpha_{I}$ must equal $\beta_{I}$. The value $\alpha_{I}$ can exist in the positive region only if the numerator and denominator of eq. (13) are both

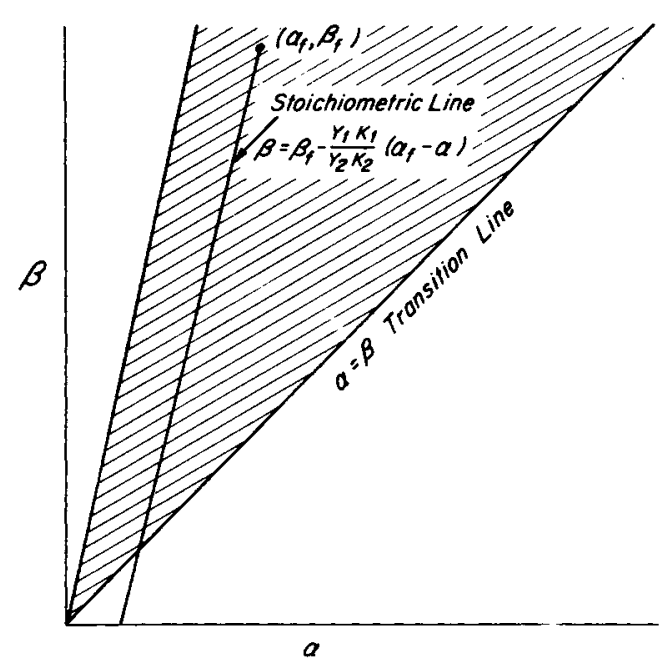

Fig. 9. Representative figure showing the general feed conditions required (shaded area) for simultaneous limitation by two substrates. Above the transition line, dimensionless substrate $\alpha$ is limiting. Below the transition line, dimensionless substrate $\beta$ is limiting. Both may affect the growth rate in the region of the transition line. 
positive or negative. Since the designation of which substrate is $S_{1}$ and $S_{2}$ is arbitrary, the selection may be made so that $Y_{1} K_{1}>Y_{2} K_{2}$. Thus, if the substrate with the greater value for $Y_{1} K_{1}$ is chosen as $S_{1}$, then both numerator and denominator in eq. (13) must be positive for the intersection point to fall in the positive region.

From eq. (13) and from Figure 9, it becomes apparent that for an intersection to occur in the positive region, the following inequality must be satisfied:

$$
Y_{1} K_{1} / Y_{2} K_{2}>\beta_{f} / \alpha_{f}>1
$$

This is in agreement with the requirements posed by Sykes, ${ }^{5}$ and is shown in Figure 9 as the shaded region. The region which satisfies inequality (14) is quite large and tends to indicate that both doublesubstrate limitation and a switch between limiting substrates is quite common. However, it should be recognized that inequality (14) only holds for kinetics models, such as the Monod and exponential models, which approach saturation $\left(\mu / \mu_{m}=1\right)$ asymptotically. The Blackman model would impose the additional limitation that the intersection with the $\alpha=\beta$ transition line must fall in the region $0<\alpha_{I}<2$. Outside this region, neither substrate is limiting and $\mu / \mu_{m}=1$.

\section{Operational Limitations on Double-Substrate Limited Growth}

From an operational or practical standpoint, certain limitations on the region where double-substrate limitation may occur should be imposed. These may be listed as:

1) There is a maximum level in the cell population, $b_{\max }$, which can occur, above which crowding and stalling effects will cause a breakdown in theory.

2) There is a minimum level in the cell population, $b_{\min }$, below which the population is both insignificant and difficult to measure with accuracy.

3) For continuous systems there is an upper limit to the dimensionless growth rate $\left(\mu / \mu_{m}\right)_{\max }$ above which the system approaches the critical dilution rate and becomes unstable to work with. For batch systems, above some value of $\left(\mu / \mu_{m}\right)_{\max }$ the organisms are basically not seriously limited by either substrate.

4) Below some minimum value, $\left(\mu / \mu_{m}\right)_{\min }$, maintenance effects start to dominate, the yield coefficients are no longer constant, and the theory breaks down.

The third limitation is rather significant. It takes into account the fact that above certain values of dimensionless substrate concentration, the organisms are actually growing at their maximum rates; 
even though kinetic models such as the Monod and exponential claim that they are not. If the stoichiometric line intersects the $\alpha=$ $\beta$ transition line above the $\left(\mu / \mu_{m}\right)_{\max }$ value, then only one substrate would ever truly limit the growth rate.

By inserting each of the four limitations into the stoichiometric equation, four separate linear relationships can be developed which form a boundary around the region where double-substrate limitation can reasonably occur. These lines are plotted in Figure 10. With the four operational limitations, the following inequalities must hold for double-substrate limitations to be feasible:

$$
\begin{aligned}
& \alpha_{I}+b_{\max } / Y_{2} K_{2}>\beta_{f}>b_{\min } / Y_{2} K_{2}+\alpha_{I} \\
& \alpha_{I_{1}}+\frac{K_{1} Y_{1}}{K_{2} Y_{2}}\left(\alpha_{f}-\alpha_{I_{1}}\right)>\beta_{f}>\frac{K_{1} Y_{1}}{K_{2} Y_{2}}\left(\alpha_{f}-\alpha_{I_{2}}\right)+\alpha_{I_{2}}
\end{aligned}
$$

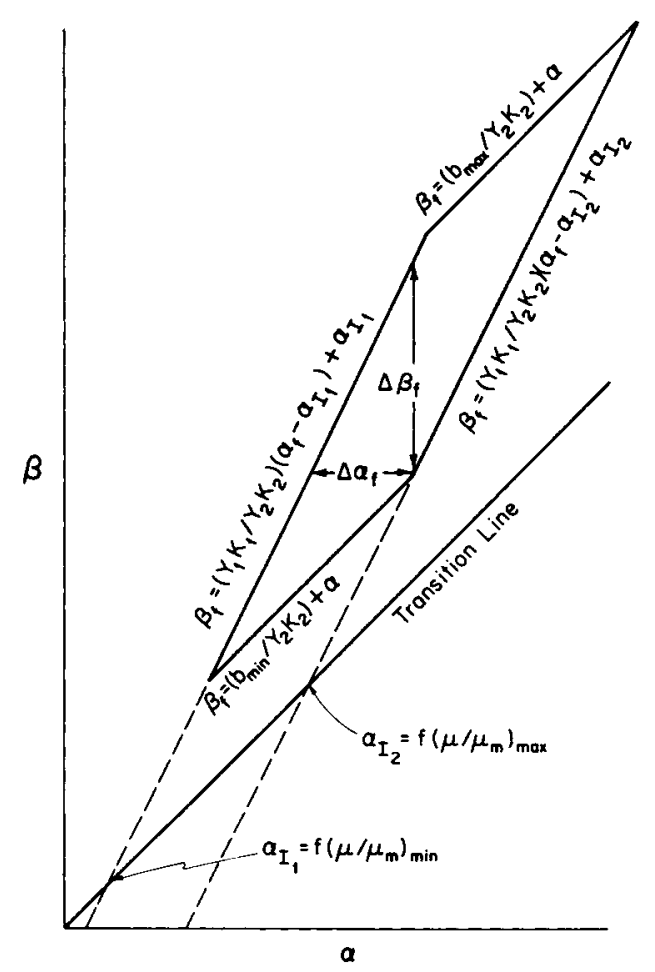

Fig. 10. Representative figure showing the operational limits on the region of feed substrate concentrations $\left(\alpha_{f}, \beta_{f}\right)$ where double-substrate limitation is likely to occur and be significant. See text for explanation. 
where $\alpha_{I}$ may be any point of intersection between $\alpha_{I_{1}}$ and $\alpha_{I_{2}}$ which are the limits imposed by the limitations of $\left(\mu / \mu_{m}\right)_{\min }$ and $\left(\mu / \mu_{m}\right)_{\max }$, respectively. The relationships between $\alpha_{I}$ and the dimensionless specific growth rate for the six models that were discussed earlier are given in Appendix III.

\section{DISCUSSION}

The region that is mapped out in Figure 10 represents the acceptable values of $\alpha_{f}$ and $\beta_{f}$ which will lead to double-substrate limitation. That is, if $\alpha_{f}$ and $\beta_{f}$ fall within the outlined region, then either or both substrates may control the growth rate of the organisms. In the case of a batch growth curve, the system would start out at a set of initial substrate concentrations $\alpha_{f}$ and $\beta_{f}$ which are within the region. As the cell population grows, the substrate concentrations ( $\alpha$ and $\beta$ ) will begin to drop, following the stoichiometric line for the particular system. Eventually it will intersect the abscissa, and no further growth will occur. Above the transition line, the growth rate will primarily be controlled by the $\alpha$ substrate concentration. Near the transition line both substrates will affect the growth rate. Below the transition line, the growth rate will primarily be controlled by the $\beta$ substrate concentration. The ultimate extent of growth would be dependent upon the $\beta$ substrate, whereas the initial growth rate would be controlled by the $\alpha$ substrate. Similar types of behavior would occur for continuous cultures.

Some estimations of the size of the double-substrate limitation region can be ascertained by determining the width $\left(\Delta \alpha_{f}\right)$ and the height $\left(\Delta \beta_{f}\right)$ of the region (see Fig. 10). Values for these parameters may be determined from:

$$
\begin{aligned}
& \Delta \alpha_{f}=\frac{K_{2} Y_{2}}{K_{1} Y_{1}}\left(\alpha_{I_{1}}-\alpha_{I_{2}}\right)+\left(\alpha_{I_{2}}-\alpha_{I_{1}}\right) \\
& \Delta \beta_{f}=\frac{K_{1} Y_{1}}{K_{2} Y_{2}}\left(\alpha_{I_{2}}-\alpha_{I_{1}}\right)+\left(\alpha_{I_{1}}-\alpha_{I_{2}}\right)
\end{aligned}
$$

as long as we are not too close to the ends of the region. These values may be useful in determining whether double-substrate limitation is likely to occur in a system. Appendix IV contains estimates of $\Delta \alpha_{f}$ and $\Delta \beta_{f}$ for two different cases of double-substrate limited growth. 
By comparing the width and height of the double-substrate limiting region to the initial concentrations of substrate which would be required, a qualitative feel for the relative possibility of operating within this region may be obtained. In the cases shown in Appendix IV, it is much more likely that double-substrate limitation would occur in case I (glucose-riboflavin system) than in case II (glucose-ammonia system).

\section{CONCLUSIONS}

The analyses which have been presented deal primarily with systems where both potentially limiting substrates are present in a single liquid phase. For growth systems, where one limiting nutrient is supplied independently from the other, the possibility of producing double-substrate limitation would be increased.

Two different philosophies exist as to the nature of growth with two limiting substrates. Both interactive and noninteractive substrate limitation would appear to be reasonable approaches. It is expected that both types exist for certain different types of substrates. A simple and unique kinetic model which handles all types of two-substrate limited growth does not appear to exist.

A method for determining the operational region where doublesubstrate limitation may occur has been presented. This method may be useful in choosing a microbial-substrate system for studying double-substrate limitation and in analyzing whether two particular substrates are likely to simultaneously limit growth in industrial processes and natural ecosystems.

APPENDIX I: SINGLE ROUTE, TWO-SUBSTRATE, ENZYME MODEL

Route I only:

$$
\frac{d P_{1}}{d t}=\frac{k_{5} E_{r} S_{1} S_{2}}{\alpha K^{\prime}{ }_{1} K_{2}+K^{\prime}{ }_{1} S_{2}+S_{1} K_{2}+S_{1} S_{2}}
$$

where $K_{1}^{\prime}=k_{5} / k_{1}$ is the half velocity constant for $S_{1} ; K_{2}=\left(k_{4}+k_{5}\right) / k_{3}$ is the half velocity constant for $S_{2}$, and $\alpha^{\prime}=k_{2} / k_{5}$.

Route 2 only:

$$
\frac{d P_{1}}{d t}=\frac{k_{5} E_{T} S_{1} S_{2}}{\alpha^{\prime} K_{2}^{\prime} K_{1}+K_{2}^{\prime} S_{1}+S_{2} K_{1}+S_{1} S_{2}}
$$

where $K_{1}=\left(k_{5}+k_{9}\right) / k_{8}$ is the half velocity constant for $S_{1} ; K_{2}^{\prime}=k_{5} / k_{6}$ is the half velocity constant for $S_{2}$, and $\alpha^{\prime \prime}=k_{7} / k_{5}$. 
Note. By analogy

$$
\frac{d P_{1}}{d t} \frac{1}{k_{5} E_{T}}=\frac{\mu}{\mu_{m}}
$$

APPENDIX II: TWO ROUTES ACTING SIMULTANEOUSLY

$$
\begin{aligned}
& A_{1}=\frac{k_{4}+k_{5}+k_{9}}{k_{8}} \quad A_{5}=\frac{k_{3} S_{2} / k_{1}}{k_{4} / k_{1}-S_{1}} \\
& A_{2}=\frac{k_{4}+k_{5}+k_{9}}{k_{3}} \quad A_{6}=\frac{S_{2}}{k_{9} / k_{6}-S_{2}} \\
& A_{3}=\frac{S_{1}}{k_{4} / k_{1}-S_{1}} \quad A_{7}=\frac{k_{7} / k_{8}}{k_{9} / k_{6}-S_{2}} \\
& A_{4}=\frac{k_{2} / k_{1}}{k_{4} / k_{1}-S_{1}} \quad A_{8}=\frac{k_{8} S_{1} / k_{6}}{k_{9} / k_{6}-S_{2}} \\
& \frac{E S_{2}}{E_{T}}=\left[\frac{S_{2} / A_{2}-A_{3}-A_{4}-A_{5}}{A_{3}-S_{1} / A_{1}} \frac{A_{3}-A_{6}}{A_{3}+A_{4}+A_{5}-A_{6}}+\left(A_{3}-\frac{S_{1}}{K_{1}}\right)\right] / \\
& \left(1-\frac{S_{2} / A_{2}-A_{3}-A_{4}-A_{5}}{A_{3}-S_{1} / A_{1}} \frac{A_{6}+A_{7}+A_{8}-A_{3}}{A_{3}+A_{4}+A_{5}-A_{6}}\right) \\
& \frac{E S_{1}}{E_{T}}=\frac{A_{6}+A_{7}+A_{8}-A_{3}}{A_{3}+A_{4}+A_{5}-A_{6}} \frac{E S_{2}}{E_{T}}+\frac{A_{2}-A_{6}}{A_{3}+A_{4}+A_{5}-A_{6}} \\
& \frac{E S_{1} S_{2}}{E_{T}}=\left(A_{6}+A_{7}+A_{8}\right) \frac{E S_{2}}{E_{T}}-A_{8}+A_{6} \frac{E S_{1}}{E_{T}} \\
& \frac{d P_{1}}{d t}=k_{5}\left(E S_{1} S_{2}\right)
\end{aligned}
$$

By analogy

$$
\frac{d P_{1}}{d t} \frac{1}{k_{5} E_{T}}=\frac{E S_{1} S_{2}}{E_{T}}=\frac{\mu}{\mu_{m}}
$$

APPENDIX III: RELATIONSHIPS BETWEEN INTERSECTS AND SPECIFIC

\section{GROWTH RATE LIMITATIONS}

Noninteractive Models

$$
\begin{array}{ccc}
\begin{array}{c}
\text { Monod } \\
\alpha_{I}=\frac{\mu / \mu_{m}}{1-\mu / \mu_{m}}
\end{array} & \alpha_{I}=\frac{1}{0.6931} \ln \frac{1}{1-\mu / \mu_{m}} & \begin{array}{c}
\text { Blackman } \\
\alpha_{I}=2 \mu / \mu_{m}
\end{array} \\
& \text { Interactive Models } & \\
\alpha_{I}=\frac{\left(\mu / \mu_{m}\right)^{1 / 2}}{1-\left(\mu / \mu_{m}\right)^{1 / 2}} & \alpha_{I}=\frac{1}{0.6931} \ln \frac{1}{1-\left(\mu / \mu_{m}\right)^{1 / 2}} & \alpha_{I}=\left(4 \mu / \mu_{m}\right)^{1 / 2}
\end{array}
$$




\section{APPENDIX IV: ESTIMATES OF THE SIZE OF A DOUBLE-SUBSTRATE LIMITED OPERATING REGION}

Assume:

$$
\begin{array}{rlrl}
\left(\frac{\mu}{\mu_{m}}\right)_{\max } & =0.9 & \left(\frac{\mu}{\mu_{m}}\right)_{\min } & =0.1 \\
b_{\max } & =10^{12} \mathrm{cell} / \mathrm{liter} \quad b_{\min }=10^{9} \mathrm{cell} / \mathrm{iter}
\end{array}
$$

Case 1, data from McGee et al. ${ }^{8}$

$$
\begin{array}{ccc}
S_{1}=\text { riboflavin }(\alpha) & K_{1}=9.37 \times 10^{-7} \mathrm{~g} / \text { liter } & Y_{1}=4.67 \times 10^{16} \mathrm{cells} / \mathrm{g} \\
S_{2}=\text { glucose }(\beta) \quad K_{2}=8.7 \times 10^{-4} \mathrm{~g} / \mathrm{liter} & Y_{2}=4.03 \times 10^{11} \mathrm{cells} / \mathrm{g} \\
K_{1} Y_{1}=4.376 \times 10^{10} \text { cells } / \text { liter } \quad K_{2} Y_{2}=3.506 \times 10^{8} \mathrm{cells} / \mathrm{iter} \\
K_{1} Y_{1} / K_{2} Y_{2}=124.81
\end{array}
$$

\begin{tabular}{ccc} 
Model & $\begin{array}{c}\text { Interactive } \\
\text { Monod }\end{array}$ & $\begin{array}{c}\text { Noninteractive } \\
\text { Blackman }\end{array}$ \\
\cline { 1 - 2 }$\alpha_{I_{1}}$ & 0.463 & 0.20 \\
$\alpha_{l_{2}}$ & 18.487 & 1.80 \\
$\Delta \alpha_{f}$ & 17.883 & 1.587 \\
$\Delta S_{1}$ & $1.68 \times 10^{-5} \mathrm{~g} /$ liter & $1.487 \times 10^{-6} \mathrm{~g} / \mathrm{liter}$ \\
$\Delta \beta_{f}$ & 2232 & 198 \\
$\Delta S_{2}$ & $1.942 \mathrm{~g} /$ liter & $0.172 \mathrm{~g} /$ liter
\end{tabular}

Note that to produce $10^{12}$ cells/liter would require roughly $2.14 \times 10^{-5} \mathrm{~g} / \mathrm{liter}$ riboflavin $\left(S_{1}\right)$ and $2.48 \mathrm{~g} /$ liter glucose $\left(S_{2}\right)$.

Case II

$$
\begin{array}{lcc}
S_{1}=\text { ammonia }(\alpha) & K_{1}=1 \times 10^{-3} \mathrm{~g} / \text { liter } & Y_{1}=4.03 \times 10^{12} \mathrm{cells} / \mathrm{g} \\
S_{2}=\text { glucose }(\beta) & K_{2}=8.7 \times 10^{-4} \mathrm{~g} / \text { liter } & Y_{2}=4.03 \times 10^{11} \mathrm{cells} / \mathrm{g} \\
& K_{1} Y_{1}=4.03 \times 10^{9} \text { cell/liter } \quad K_{2} Y_{2}=3.506 \times 10^{8} \text { cell/liter } \\
& K_{1} Y_{1} / K_{2} Y_{2}=11.494 & \\
& \text { Interactive } & \text { Noninteractive } \\
\text { Model } & \text { Monod } & \text { Blackman } \\
\hline \alpha_{I_{1}} & 0.463 & 0.2 \\
\alpha_{I_{2}} & 18.487 & 1.80 \\
\Delta \alpha_{f} & 16.456 & 1.461 \\
\Delta S_{1} & 1.65 \times 10^{-2} \mathrm{~g} / \text { liter } & 1.461 \times 10^{-3} \mathrm{~g} / \text { liter } \\
\Delta \beta_{f} & 189.14 & 16.79 \\
\Delta S_{2} & 0.1646 \mathrm{~g} / \text { liter } & 0.0146 \mathrm{~g} / \mathrm{liter}
\end{array}
$$

$\frac{\text { Model }}{\alpha_{I_{1}}}$

0.2
1.80
1.461
$1.461 \times 10^{-3} \mathrm{~g} /$ liter
16.79
$0.0146 \mathrm{~g} /$ liter

To produce $10^{12}$ cells/liter would require roughly 0.248 g/liter ammonia $\left(S_{1}\right)$ and 2.48 g/liter glucose $\left(S_{2}\right)$. 


\section{Nomenclature}

$\begin{array}{ll}b & \text { bacterial concentration } \\ E & \text { free enzyme concentration } \\ E_{T} & \text { total enzyme concentration, all forms } \\ \left(E S_{i}\right) & \text { enzyme- } S_{i} \text { complex concentration } \\ k_{i} & \text { rate constants } \\ K_{i}, K^{\prime}{ }_{i} & \text { half velocity constants for substrate } i \\ P_{i} & \text { product concentration } \\ S_{i} & \text { substrate concentration } \\ t & \text { time } \\ X_{i} & \text { equilibrium constant for reaction } i \text { in Figure } 6 \\ Y_{i} & \text { yield coefficient for substrate } i \\ \alpha^{\prime}, \alpha^{\prime \prime} & \text { single route-two-substrate enzyme model constants defined in Ap- } \\ \alpha, \beta & \text { pendix I } \\ \theta & \text { dimensionless substrate concentrations, } S / K \\ \mu, \mu_{m} & \text { holding time for continuous culture } \\ & \text { specific and maximum specific growth rates of organisms, } \mathrm{hr}^{-1}\end{array}$

\section{Scripts}

$f \quad$ feed concentration

tilde; steady-state values

\section{References}

1. F. F. Blackman, Ann. Bot., 19, 281 (1905).

2. F. G. Bader, J. S. Meyer, A. G. Fredrickson, and H. M. Tsuchiya, Biotechnol. Bioeng., 17, 279 (1975).

3. J. Monod, Ann. Rev. Microbiol, 3, 371 (1949).

4. J. N. Dabes, R. K. Finn, and C. R. Wilke, Biotechnol. Bioeng., 15, 1159 (1973).

5. R. M. Sykes, J. Water Pollut. Control Fed., 45, 888 (1973).

6. M. R. Droop, J. Mar. Biol. Ass. U.K., 54, 825 (1974).

7. D. N. Ryder and C. G. Sinclair, Biotechnol. Bioeng., 14, 787 (1972).

8. R. D. McGee, J. F. Drake, A. G. Fredrickson, and H. M. Tsuchiya, Can. J. Microbiol., 18, 1733 (1972).

9. J. A. Howell and B. Atkinson, Biotechnol. Bioeng., 18, 15 (1976).

Accepted for Publication June 30, 1977 